\title{
A NEW CHARACTERIZATION OF THE $F$ SET OF A RATIONAL FUNCTION $\left({ }^{1}\right)$
}

\author{
BY
}

\author{
MARILYN K. OBA AND TOM S. PITCHER
}

\begin{abstract}
In the early part of this century G. Julia and P. Fatou extensively studied the iteration of functions on the complex plane. More recently Hans Brolin reopened the investigation. In this paper, we are interested in the $F$ set which is the set of points at which the family of iterates of a given rational function $R$ is not normal and in a measure which is in some sense naturally imposed on the $F$ set by the iterates of $R$.

We construct a sequence of probability measures via the inverse functions of the iterates of $R$ and almost any starting point. The measure of primary interest is the weak limit of such sequences. These weak limits are supported by $F$ and have certain invariance properties. We establish that this weak limit measure is unique and is ergodic with respect to the transformation $R$ on the $F$ set for a large class of rational functions. In the course of the proof of uniqueness we develop expressions for the logarithmic potential function and for the energy integral of $F$. We also establish inequalities for the capacity of the $F$ set which become equalities for the polynomial case.
\end{abstract}

0. Preliminaries. Let $R(z)=P(z) / Q(z)$ be a rational function of degree $N, N$ at least two, where $P$ is a monic polynomial and $Q$ a polynomial. We also assume that $P$ and $Q$ have no common roots. The sequence of iterates $\left\{R_{n}(z)\right\}$ is defined by

$$
R_{0}(z)=z, \quad R_{1}(z)=R(z), \quad R_{n+1}(z)=R\left(R_{n}(z)\right) \quad n=1,2, \ldots,
$$

where $z$ takes on values from the extended complex plane, $\hat{C}$, with its topology as the compactified Riemann sphere.

If $w=R_{n}(z)$ we say that $w$ is a successor of $z$ and $z$ is a predecessor of $w$, in both cases of order $n$. If $w=R_{n}(w)$ and $R_{p}(w) \neq w$ when $p<n$, we say that $w$ is a fixpoint of order $n$. The derivative $R_{n}^{\prime}(w)$ is called the multiplier of $w$. The successor of a fixpoint of order $n$ is a fixpoint of order $n$. Furthermore, the set $\left\{w, R(w), R_{2}(w)\right.$, $\left.\ldots, R_{n-1}(w)\right\}$ is called a cycle of order $n$, and all fixpoints of an $n$-cycle have the same multiplier since $R_{n}^{\prime}(w)=\prod_{k=0}^{n-1} R^{\prime}\left(R_{k}(w)\right)$. A fixpoint $w$ (or a cycle) of order $n$ is called attractive, indifferent or repulsive according as $\left|R_{k}^{\prime}(w)\right|<1,=1$, or $>1$ respectively.

The set $F$ consists of those points at which the sequence $\left\{R_{n}(z)\right\}$ is not normal in the sense of Montel (see Fatou [2]). An alternate characterization for $F$ is the

Received by the editors March 17, 1971 and, in revised form, August 6, 1971.

AMS 1970 subject classifications. Primary 30A20; Secondary 30A44.

Key words and phrases. Iteration of rational functions, $F$ set, weak limit measure, ergodicity, logarithmic capacity.

(1) Work supported by National Science Foundation grant GP-21331.

Copyright (C) 1972, American Mathematical Society 
closure of the set of repulsive fixpoints. (See Brolin [1, Theorem 4.1, p. 109], or Julia [4, p. 48].) We shall use the first characterization. The reader may refer to [1] for the results of this section and for the monic polynomial case of §III.

One easily sees from the definition of $F$ that $F$ is closed and completely invariant under $R$, i.e., $R_{-1}(F)=F=R(F)$.

I. The exceptional points. For the rest of the paper we shall assume that the degree of $P$ is greater than the degree of $Q$. In this case the point at infinity will be an attractive fixpoint if and only if the coefficient of $z^{N-1}$ in $Q$ has absolute value less than one. Since attractive fixpoints are outside the $F$ set it will then be compact; in particular, this is the case when the degree of $P$ exceeds the degree of $Q$ by at least two. In general, the $F$ set is a compact subset of the Riemann sphere.

We shall call a cycle $\left\{z_{0}, z_{1}, \ldots, z_{m}\right\}$ a maximal cycle if $R(z)=z_{i}$ has $z_{i-1}$ as its only solution, and $R(z)=z_{0}$ has $z_{m}$ as its only solution. We shall see later that $m=0$ or 1.

By Montel's theorem, in every neighborhood of $w \in F$ the functions $R_{n}$ omit at most two values. These, if they occur, are called exceptional points. We next characterize the exceptional points as points of maximal cycles. Such points will be of importance in what follows.

THEOREM 1. A point belongs to a maximal cycle if and only if it is axceptional point. Such points are attractive fixpoints and consequently not in $F$.

Proof. Points in maximal cycles are attractive fixpoints, in fact, $R^{\prime}\left(z_{0}\right)=0$ if $z_{0}$ belongs to a maximal cycle. Let $z_{0}$ and $z_{1}$ be consecutive points in a maximal cycle; $z_{0}$ is not necessarily different from $z_{1}$. Then the equation $R(z)-z_{1}=0$ leads us to the equation $P(z)=\left(z-z_{0}\right)^{N}-z_{1} Q(z)$. We have obtained

and

$$
R(z)=\left(z-z_{0}\right)^{N} / Q(z)-z_{1}
$$

$$
R^{\prime}(z)=\left[N\left(z-z_{0}\right)^{N-1} Q(z)-\left(z-z_{0}\right)^{N} Q^{\prime}(z)\right] / Q^{2}(z),
$$

so $\left|R^{\prime}\left(z_{0}\right)\right|=0$. In order for $\infty$ to belong to a maximal cycle, $R(z)$ must either be a polynomial or $R(z)=1 / c z^{N}$. In the first case $\infty$ is an attractive fixpoint of order one; the second case is outside the class under consideration. Hence any point of a maximal cycle is an attractive fixpoint, and thus belongs to the complement of $F$.

Now suppose $z_{0}$ belongs to a maximal cycle $\left\{z_{0}, z_{1}, \ldots, z_{m}\right\}$. Let $w$ be any point in $F$, and let $U$ be any neighborhood of $w$ not intersecting the maximal cycle, then $\left\{R_{n}(z)\right\} \cap\left\{z_{0}, z_{1}, \ldots, z_{m}\right\}=\varnothing$ for any $n$ and any $z$ in $U$ by the nature of a maximal cycle. Hence, $z_{0}$ and, in fact, all the points of the maximal cycle are exceptional points.

On the other hand, let $z_{0}$ be an exceptional point. $z_{0}$ may be the only exceptional point and consequently its only predecessor and successor, in which case $z_{0}$ is a maximal one-cycle. The other possibility is the existence of two exceptional points $z_{0}$ and $z_{1}$, so that $z_{0}$ may have the point $z_{1}$ as a predecessor. 
In this latter case, consideration of the equations $R(z)-z_{0}=0$ and $R(z)-z_{1}=0$ where $R(z)=P(z) / Q(z)$ leads to

$$
\begin{aligned}
& P(z)-z_{0} Q(z)=\left(z-z_{0}\right)^{n}\left(z-z_{1}\right)^{N-n} \\
& P(z)-z_{1} Q(z)=\left(z-z_{0}\right)^{m}\left(z-z_{1}\right)^{N-m} .
\end{aligned}
$$

Now suppose $n<m$, then subtracting the two equations above,

$$
Q(z)=\left(z_{1}-z_{0}\right)^{-1}\left[\left(z-z_{0}\right)^{n}\left(z-z_{1}\right)^{N-m}\right]\left[\left(z-z_{1}\right)^{m-n}-\left(z-z_{0}\right)^{m-n}\right] .
$$

Multiplying the first equation by $z_{1}$ and the second by $z_{0}$, then subtracting, we obtain

$$
P(z)=\left(z_{1}-z_{0}\right)^{-1}\left[\left(z-z_{0}\right)^{n}\left(z-z_{1}\right)^{N-m}\right]\left[z_{1}\left(z-z_{1}\right)^{m-n}-z_{0}\left(z-z_{0}\right)^{m-n}\right] .
$$

Since $P$ and $Q$ have no common factors, these equations imply that $n=0$ and $m=N$, thus $P(z)-z_{0} Q(z)=\left(z-z_{1}\right)^{N}$. In this instance $\left\{z_{0}, z_{1}\right\}$ must be a maximal two-cycle.

Supposing instead that $m<n$, a similar computation shows that $\left\{z_{0}\right\}$ is a maximal one-cycle.

The assumption $n=m$ leads us to $z_{0}=z_{1}$ or the first case where $z_{0}$ is the only exceptional point and hence a maximal one-cycle. We conclude that the exceptional points are points of maximal cycles.

COROllary. A maximal cycle is at most of length two.

THEOREM 2. We have $R(z)=P(z) / Q(z)$ where $P$ and $Q$ are polynomials, $P$ with leading coefficient one. Suppose that the degree of $P=N>$ degree of $Q$ and $N \geqq 2$. We can characterize the occurrence of maximal cycles, i.e., exceptional points, as follows:

(i) degree of $Q=0:$ In this case $\{\infty\}$ is a maximal one-cycle. There may be no other one-cycles or one other finite one-cycle, say $\left\{z_{0}\right\}$. In the latter case $P(z)$ $=\left(z-z_{0}\right)^{N}+z_{0} Q$.

(ii) degree of $Q>0$ : There may be one finite maximal one-cycle $\left\{z_{0}\right\}$ in which event $P(z)=\left(z-z_{0}\right)^{N}+z_{0} Q(z)$. Secondly, we may have two finite maximal one-cycles $\left\{z_{0}\right\}$ and $\left\{z_{1}\right\}$, then

$$
\begin{aligned}
& P(z)=\left(z_{1}-z_{0}\right)^{-1}\left[z_{1}\left(z-z_{0}\right)^{N}-z_{0}\left(z-z_{1}\right)^{N}\right] \\
& Q(z)=\left(z_{1}-z_{0}\right)^{-1}\left[\left(z-z_{0}\right)^{N}-\left(z-z_{1}\right)^{N}\right] .
\end{aligned}
$$

Thirdly, $R(z)$ may produce a single maximal two-cycle $\left\{z_{0}, z_{1}\right\}$ in which case

$$
\begin{aligned}
& P(z)=\left(z_{1}-z_{0}\right)^{-1}\left[z_{1}\left(z-z_{1}\right)^{N}-z_{0}\left(z-z_{0}\right)^{N}\right], \\
& Q(z)=\left(z_{1}-z_{0}\right)^{-1}\left[\left(z-z_{1}\right)^{N}-\left(z-z_{0}\right)^{N}\right] .
\end{aligned}
$$

Proof. (i) $R(z)$ is a polynomial so $\{\infty\}$ is a maximal one-cycle. Suppose we have a finite maximal one-cycle $\left\{z_{0}\right\}$. Since $z_{0}$ is the only solution of $R(z)-z_{0}=0$, we have $P(z)-z_{0} Q=\left(z-z_{0}\right)^{N}$. 
(ii) We suppose that the degree of $Q>0$. Again suppose we have only one finite maximal one-cycle $\left\{z_{0}\right\}$. In this case $P(z)-z_{0} Q(z)=\left(z-z_{0}\right)^{N}$ so $P$ takes the form $P(z)=\left(z-z_{0}\right)^{N}+z_{0} Q(z)$.

Suppose we have two maximal one-cycles $\left\{z_{0}\right\}$ and $\left\{z_{1}\right\}$. Then $R(z)-z_{0}=0$ has one solution $z_{0}$, and $R(z)-z_{1}=0$ has one solution $z_{1}$, so $P(z)-z_{0} Q(z)=\left(z-z_{0}\right)^{N}$ and $P(z)-z_{1} Q(z)=\left(z-z_{1}\right)^{N}$, or

$$
P(z)=\left(z-z_{0}\right)^{N}+z_{0} Q(z) \text { and } P(z)=\left(z-z_{1}\right)^{N}+z_{1} Q(z) .
$$

Subtracting, we obtain

$$
Q(z)=\left(z_{1}-z_{0}\right)^{-1}\left[\left(z-z_{0}\right)^{N}-\left(z-z_{1}\right)^{N}\right] .
$$

Thus

$$
\begin{aligned}
P(z) & =\left(z-z_{0}\right)^{N}+\left(z_{0} /\left(z_{1}-z_{0}\right)\right)\left[\left(z-z_{0}\right)^{N}-\left(z-z_{1}\right)^{N}\right] \\
& =\left(z_{1}-z_{0}\right)^{-1}\left[z_{1}\left(z-z_{0}\right)^{N}-z_{0}\left(z-z_{1}\right)^{N}\right] .
\end{aligned}
$$

Finally, we consider the case of maximal cycles of length two. Let the maximal cycle be $\left\{z_{0}, z_{1}\right\}$. From the equations $P(z)-z_{0} Q(z)=\left(z-z_{1}\right)^{N}$ and $P(z)-z_{1} Q(z)$ $=\left(z-z_{0}\right)^{N}$ we obtain

$$
\begin{aligned}
& P(z)=\left(z_{1}-z_{0}\right)^{-1}\left[z_{1}\left(z-z_{1}\right)^{N}-z_{0}\left(z-z_{0}\right)^{N}\right], \\
& Q(z)=\left(z_{1}-z_{0}\right)^{-1}\left[\left(z-z_{1}\right)^{N}-\left(z-z_{0}\right)^{N}\right] .
\end{aligned}
$$

II. The weak limit measure and its support $F$. The results of this section extend the results of Brolin [1] which treat the monic polynomial case. This section and the next provide a new characterization of the $F$ set as the support of a particular measure, the weak limit measure.

LEMMA 1. Given that $P$ and $Q$ have no common roots, then $P_{k}$ and $Q_{k}$ also have no common roots for all $k$. We have the following expressions for the iterates of $R$ :

$$
R=\frac{P}{Q}, \quad R_{2}=\frac{Q^{N} \cdot P \circ R}{Q^{N} \cdot Q \circ R}, \quad \ldots, \quad R_{k+1}=\frac{Q^{N^{k}} \cdot P_{k} \circ R}{Q^{N^{k}} \cdot Q_{k} \circ R}=\frac{P_{k+1}}{Q_{k+1}} .
$$

Proof. By hypothesis $P_{1}$ and $Q_{1}$ have no common roots. Let us suppose now that $P_{n}$ and $Q_{n}$ have no common roots and consider $P_{n+1}$ and $Q_{n+1}$ :

$$
P_{n+1}=Q^{N^{n}} \cdot P_{n}(P / Q) \text { and } Q_{n+1}=Q^{N^{n}} \cdot Q_{n}(P / Q) \text {. }
$$

If $w$ is a common root of $P_{n+1}$ and $Q_{n+1}$ we have

$$
Q^{N^{n}}(w) \cdot P_{n}(R(w))=0 \text { and } Q^{N^{n}}(w) \cdot Q_{n}(R(w))=0 .
$$

Since $P_{n}$ and $Q_{n}$ cannot have $R(w)$ as a common root, these equations imply that $Q^{N^{n}}(w)=0$; i.e., $Q(w)=0$. But then

$$
\begin{aligned}
0 & =Q^{N^{n}}(w) P_{n}(P(w) / Q(w)) \\
& =P(w)^{N^{n}}+a P(w)^{N^{n}-1} Q(w)+\cdots=(P(w))^{N^{n}}
\end{aligned}
$$

which contradicts the hypothesis. 
We now wish to construct a measure on the extended complex plane. Let $z_{0}$ be a point which does not belong to a maximal cycle. Define $\left\{\mu_{n}\right\}$ to be the following sequence of probability measures on the Borel $\sigma$-field, $\mathscr{B}$.

$\mu_{0}$ places weight 1 at $z_{0}$.

$\mu_{1}$ places weight $N^{-1}$ at the $N$ predecessors of order 1 of $z_{0}$. $\vdots$

$\mu_{n}$ places weight $N^{-n}$ at the $N^{n}$ predecessors of order 1 of $z_{0}$.

The weights are placed at each predecessor according to its multiplicity as a zero of $R_{n}(z)-z_{0}=0$ and Lemma 1 assures us that this equation has exactly $N^{n}$ solutions counted according to their multiplicities.

Consider the following sequence of probability measures:

$$
\nu_{n}=\frac{1}{n+1} \sum_{i=0}^{n} \mu_{i}, \quad n=1,2, \ldots
$$

This sequence of probability measures on the compactified Riemann sphere has a subsequence $\left\{v_{n_{k}}\right\}$ tending to a weak limit probability measure $\mu$, i.e., $\int f d v_{n_{k}} \rightarrow \int f d \mu$ as $k \rightarrow \infty$ for all continuous $f$.

For arbitrary $f$, set $A f(z)=N^{-1} \sum_{(w: R(w)=z)} f(w)$.

LEMMA 2. $\int A f d \mu=\int f d \mu$ for any integrable Borel measurable $f$.

Proof. For continuous $f$

$$
\begin{aligned}
\int A f d \mu & =\lim _{k \rightarrow \infty} \frac{1}{n_{k}+1} \sum_{i=0}^{n_{k}} \int A f d \mu_{i} \\
& =\lim _{k \rightarrow \infty} \frac{1}{n_{k}+1} \sum_{i=0}^{n_{k}} \frac{1}{N^{i}} \sum_{R_{i}(z)=z_{0}} A f(z) \\
& =\lim _{k \rightarrow \infty} \frac{1}{n_{k}+1} \sum_{i=0}^{n_{k}} \int f d \mu_{i+1}=\int f d \mu .
\end{aligned}
$$

The result is now extended to $\chi_{0}$ for 0 open by using a sequence $f_{n}$ of continuous functions with $f_{n} \uparrow \chi_{0}$. The sets $B$ for which $\int A \chi_{B} d \mu=\int \chi_{B} d \mu$ are easily seen to form a $\sigma$-algebra so that all Borel sets have the property. The extension to Borel measurable functions is done in the usual way.

LEMMA 3. If $\omega$ is any A-invariant probability measure, $f$ and $g$ are Borel measurable and $B$ is a Borel set, then

(i) $\int(A f) \cdot g d \omega=\int f(g \circ R) d \omega$,

(ii) $\int g d \omega=\int g \circ R d \omega$,

(iii) $\omega\left(R^{-1}(B)\right)=\omega(B)$,

(iv) $\omega(R(B)) \leqq N \omega(B)$.

((i) and (ii) hold whenever the integrals involved exist.) 
Proof. An easy computation yields $A(f \cdot g \circ R)=(A f) \cdot g$ and (i), (ii) and (iii) follow from this. Since $A \chi_{B}(z) \geqq N^{-1} \chi_{R(B)}(z)$ we have

$$
\omega(B)=\int \chi_{B} d \omega=\int A\left(\chi_{B}\right) d \omega \geqq N^{-1} \omega(R(B)) .
$$

Lemma 4. An A-invariant probability measure $\omega$ can have atoms only on points of a maximal cycle.

Proof. Suppose that $\omega(\{\zeta\})=\alpha_{0}>0$. Using Lemma 2,

$$
\begin{aligned}
\alpha_{0} & =\int \chi_{(\zeta)}(z) d \omega(z)=N^{-1} \int \sum_{R(w)=z} \chi_{(\zeta)}(w) d \omega(z) \\
& =N^{-1} \int\left[\sum_{R(w)=R(\zeta)} \chi_{\{(\zeta)}(w)\right] \cdot \chi_{\{R(\zeta)\}}(z) d \omega(z) \\
& =k_{1} N^{-1} \omega(\{R(\zeta)\})
\end{aligned}
$$

where $k_{1}$ is the multiplicity of $\zeta$ as a solution to $R(w)=R(\zeta) ; k_{1} \leqq N$. Hence $\alpha_{1}$ $=\omega(\{R(\zeta)\})=N_{1}^{-1} \alpha_{0} \geqq \alpha_{0}$. Similarly, $\alpha_{2}=\omega\left(\left\{R_{2}(\zeta)\right\}\right)=N k_{2}^{-1} \alpha_{0} \geqq \alpha_{1} \geqq \alpha_{0}$ where $k_{2}$ is the multiplicity of $R(\zeta)$ as a solution to $R(z)=R_{2}(\zeta)$, etc.

In order that $\omega$ not exceed one in total weight, $\zeta$ must be a fixpoint of some order, say $n$. So we have a cycle $\left\{\zeta, R(\zeta), \ldots, R_{n-1}(\zeta)\right\}$ with respective weights $\alpha_{0}, \alpha_{1}, \ldots, \alpha_{n-1}$. Now the monotonicity of the $\alpha_{i}$ 's forces them all to be equal since $\alpha_{0} \leqq \alpha_{1} \leqq \cdots \leqq \alpha_{n-1} \leqq \alpha_{0}$. Consequently, each $k_{i}=N$, and the cycle is a maximal cycle.

LEMMA 5. $\mu$ is nonatomic.

Proof. Suppose $\zeta$ belongs to a maximal cycle, by Lemma 4 , these are the only points at which $\mu$ can have an atom. If $\zeta$ belongs to a maximal cycle of length one, let $U$ be an open neighborhood of $\zeta$ such that $R(U) \subseteq U$ and $z_{0} \notin U$. This is possible since $\zeta$ is an attractive fixpoint of order one. If $\zeta$ and $\zeta^{\prime}$ constitute a maximal twocycle, each is an attractive fixpoint of order two. Let $U^{\prime}$ be a small neighborhood of $\zeta^{\prime}$ excluding $z_{0}$. Pick $U$ to be an open set containing $\zeta$ such that $R_{2}(U) \subseteq U$ and $R(U) \subseteq U^{\prime}, U$ also excluding $z_{0}$, then $\bigcup_{n=0}^{\infty} R_{n}(U) \subseteq U \cup U^{\prime}$.

In both cases, it is easily seen that $\mu_{n}(U)=0$ for all $n$ and hence $\mu(U)=0$.

THEOREM 3. $F$ is the support of $\mu$.

Proof. If $\zeta \in F^{c}$ there is a neighborhood $U$ of $\zeta$ such that $\left\{R_{n}\right\}$ is normal in $U$. Let $\mathcal{O}_{\zeta}$ be a subset of $U$ which is a relatively compact, open neighborhood of $\zeta$ and a $\mu$-continuity set (i.e., $\left.\left(\partial \mathcal{O}_{\zeta}\right)=0\right)$ so that $\mu\left(\mathcal{O}_{\zeta}\right)=\lim \nu_{n_{j}}\left(\mathcal{O}_{\zeta}\right)$. Then if $\zeta$ belongs to the support of $\mu$, there is a sequence $\left\{n_{k}\right\}$ such that $\mu_{n_{k}}\left(\mathcal{O}_{\zeta}\right)=N^{-k}$ (number of zeros of $R_{n_{k}}(z)-z_{0}$ in $\left.\mathcal{O}_{\zeta}\right) \geqq \varepsilon>0$. This implies that the number of zeros of $R_{n_{k}}(z)-z_{0}$ in $\mathcal{O}_{\zeta}$ approaches $\infty$ as $k \rightarrow \infty$.

Since $\left\{R_{n}\right\}$ is normal in $U$, we may extract a subsequence of $\left\{n_{k}\right\}$ which converges uniformly in compact subsets of $U$. We shall retain the notation $\left\{\boldsymbol{n}_{k}\right\}$ for such a 
subsequence. Then $R_{n_{k}}$ converges uniformly to some meromorphic $L$ on $\mathcal{O}_{\zeta}$. If $L$ is not constant there is a small circle about $\zeta$ where $L(w)-z_{0}$ is bounded away from 0. By Rouché's theorem, for large enough $k, L(w)-z_{0}$ and $R_{n_{k}}(w)-z_{0}$ must have the same number of zeros, i.e., $L(w) \equiv z_{0}$.

Now let $f$ be any function such that $f$ is continuous, $f\left(z_{0}\right)=1$ and $f \geqq 0$. Then

$$
\int f d \mu=\int f \circ R_{n_{k}} d \mu \geqq \int_{\mathcal{O}_{\zeta}} f \circ R_{n_{k}} d \mu \rightarrow f\left(z_{0}\right) \mu\left(\mathcal{C}_{\zeta}\right)=\mu\left(\mathcal{O}_{\zeta}\right)>0 .
$$

Thus $\mu$ must have an atom at $z_{0}$, which is impossible, so the support of $\mu \subseteq F$.

To show the reverse inclusion, suppose $\zeta \in F$ and there is a neighborhood $\mathcal{O}$ containing $\zeta$ with $\mu(\mathcal{O})=0$. By Lemma $3, \mu\left(R_{n}(\mathcal{O})\right)=0, n=1,2, \ldots$, so that $\mu(\hat{C}-\{$ exceptional points $\})=\left(\bigcup_{n=0}^{\infty} R_{n}(\mathcal{O})\right)=0$ which would imply that $\mu$ was atomic.

III. The uniqueness and ergodicity of the weak limit measure. Let $\mathscr{F}$ be a countable set of continuous functions dense in the set of continuous functions on $\hat{C}$ in the uniform norm sense. Let

$$
C_{f}=\left\{z: \frac{1}{n+1} \sum_{k=0}^{n} A^{k} f(z) \text { converges as } n \rightarrow \infty\right\}
$$

for each $f$ in $\mathscr{F}$. By the ergodic theorem of Hopf, $\mu\left(C_{f}\right)=1$ for any $A$-invariant probability measure $\mu$. Let $\mathscr{C}=\bigcap_{f \in \mathscr{F}} C_{f}-$ exceptional points $\}$, then $\mu(\mathscr{C})=1$ for any nonatomic $A$-invariant probability measure.

LEMMA 6. For any $z$ in $\mathscr{C}$ and any continuous function $f$ on $\hat{C}$ we have that

$$
\frac{1}{n+1} \sum_{k=0}^{n} A^{k} f(z)=\int f d \nu_{n, z} \rightarrow \int f d \mu_{z} \text { as } n \rightarrow \infty
$$

where $\mu_{z}$ is the measure constructed above using $z$ as a starting point.

Proof. Trivial.

Set

$$
f(z)=\lim _{n \rightarrow \infty} \frac{1}{n+1} \sum_{k=0}^{n} A^{k} f(z) .
$$

This limit exists for all $z$ in $\mathscr{C}$ and all continuous $f$ by Lemma 6. If $\mu$ is any $A$ invariant measure this limit exists $\mu$-a.e. for any $f$ belonging to $L_{1}(F, \mathscr{B}, \mu)$.

LEMMA 7. For $\omega$ any nonatomic A-invariant probability measure and any continuous $f$ we have

$$
\int f d \omega=\int f d \omega=\int\left(\int f d \mu_{z}\right) d \omega .
$$

Proof. By the dominated convergence theorem

$$
\int f d \omega=\int\left(\frac{1}{n+1} \sum_{k=0}^{n} A^{k} f\right) d \omega \rightarrow \int f d \omega .
$$


We wish now to show that there is only one $A$-invariant nonatomic probability measure $\mu$ and to compute its logarithmic potential function

and its energy integral

$$
u(z)=\int \log |z-w|^{-1} d \mu(w)
$$

$$
I(\mu)=\iint \log |z-w|^{-1} d \mu(z) d \mu(w) .
$$

LeMmA 8. Suppose $Q(z)=c \prod_{j=1}^{M}\left(z-q_{j}\right)$, then

$$
A \log |Q|(z)=\log |c|+\frac{1}{N} \sum_{j=1}^{M} \log \left|P\left(q_{j}\right)\right| .
$$

Proof.

$$
\begin{aligned}
A \log |Q|(z) & =\frac{1}{N} \sum_{R(w)=z} \log |Q(w)|=\frac{1}{N} \log \left|c^{N} \prod_{j=1}^{M} \prod_{R(w)=z}\left(w-q_{j}\right)\right| \\
& =\log |c|+\frac{1}{N} \sum_{j=1}^{M} \log \left|P\left(q_{j}\right)-z Q\left(q_{j}\right)\right| \\
& =\log |c|+\frac{1}{N} \sum_{j=1}^{M} \log \left|P\left(q_{j}\right)\right| .
\end{aligned}
$$

We have used here the fact that $\prod_{R(w)=z}(q-w)=P(q)-z Q(q)$.

THEOREM 4. For $R(z)=P(z) / Q(z), P$ and $Q$ polynomials with the leading coefficient of $P$ equal to one and the degree of $P=N \geqq$ the degree of $Q+2$, the weak limit measure $\mu_{z}$ is unique; i.e., $\mu_{z}$ is independent of $z$ where $z$ is any nonexceptional point. In fact, all nonatomic A-invariant probability measures are identical. Furthermore, the logarithmic potential function and the energy integral of this $\mu$ are given by

$$
\begin{aligned}
u(w) & =-\frac{1}{N} \sum_{j=0}^{\infty} N^{-j} \log \left|Q \circ R_{j}(w)\right| \text { for any } w \text { in } F, \\
I(\mu) & =\frac{1}{1-N} \int \log |Q(w)| d \mu(w) \\
& =\frac{1}{1-N} \log |c|+\frac{1}{N(1-N)} \sum_{j=1}^{M} \log \left|P\left(q_{j}\right)\right|
\end{aligned}
$$

where $Q(z)=c \prod_{j=1}^{M}\left(z-q_{j}\right)$.

Proof. We first need the formula for the logarithmic potential function. For $\zeta$ in $C$ we have

$$
\begin{aligned}
u(w) & =\int \log \frac{1}{|w-z|} d \mu_{\zeta}(z)=\int A^{k} \log \frac{1}{|w-z|} d \mu_{\zeta}(z) \\
& =\int N^{-k}\left(\sum_{R_{k}(\eta)=z} \log \frac{1}{|\eta-w|}\right) d \mu_{\zeta}(z) \\
& =\int N^{-k}\left(\log \frac{1}{\left|R_{k}(w)-z\right|}+\log \frac{1}{\left|Q_{k}(w)\right|}\right) d \mu_{\zeta}(z)
\end{aligned}
$$


If $w \in F, \log \left|R_{k}(w)-z\right|^{-1} \geqq \log (\text { diameter of } F)^{-1}$ for $z \in F$, so

$$
\begin{aligned}
u(w) & \geqq \lim _{k \rightarrow \infty} N^{-k} \int \log \frac{1}{\left|Q_{k}(w)\right|} d \mu_{\zeta}(z) \\
& =\lim _{k \rightarrow \infty}-N^{-k} \log \left|Q_{k}(w)\right| \\
& =\lim _{k \rightarrow \infty}-\left(N^{-k} \log |Q|^{N^{k-1}}+N^{-k} \log \left|Q_{k-1} \circ R\right|\right)(w) \\
& =\cdots=-\frac{1}{N} \sum_{j=0}^{\infty} N^{-j} \log \left|Q \circ R_{j}(w)\right| .
\end{aligned}
$$

Now for arbitrary $w$, if $R_{k}(w) \rightarrow \infty$ then $\log \left|R_{k}(w)-z\right|^{-1} \rightarrow-\infty$ for all $z$ in $F$, so by a similar computation

$$
u(w) \leqq-\frac{1}{N} \sum_{j=0}^{\infty} N^{-j} \log \left|Q \circ R_{j}(w)\right| .
$$

If $w_{k} \rightarrow w \in F$, and $\lim _{j \rightarrow \infty}\left|R_{j}\left(w_{k}\right)\right|=\infty$, then

$$
\begin{aligned}
u(w) & =\lim _{T \rightarrow \infty} \int \min \left(\log \frac{1}{|w-z|}, T\right) d \mu_{\zeta} \\
& =\lim _{T \rightarrow \infty} \lim _{k \rightarrow \infty} \int \min \left(\log \frac{1}{\left|w_{k}-z\right|}, T\right) d \mu_{\zeta} \\
& \leqq \liminf _{k \rightarrow \infty}-\frac{1}{N} \sum_{j=0}^{\infty} N^{-j} \log \left|Q \circ R_{j}\left(w_{k}\right)\right| .
\end{aligned}
$$

It remains to find a sequence $\left\{w_{k}\right\}$ for which the last limit can be taken term by term. If $M=0$ so that $Q$ is constant, this can easily be done. Otherwise set $L=N-M$ and proceed as follows: for large enough $a,|z| \geqq a$ implies

(i) $|R(z)|>2|z|$,

(ii) $|R(z)| \leqq b|z|^{L}$,

(iii) $|Q(z)| \leqq b|z|^{M}$, and

(iv) $|Q(z)| \geqq 1$

where $b$ is some constant. For $w$ in $F$ we can find a sequence $\left\{w_{k}\right\}$ converging to $w$ such that for each $w_{k}$ there is an $l=l(k)$ with $\left|R_{j}\left(w_{k}\right)\right|<a$ for $j<l$ and $a \leqq\left|R_{l}\left(w_{k}\right)\right|$ $<2 a$, since otherwise there would be a neighborhood $V$ of $w$ for which $R_{j}(V)$ missed the entire annulus $a \leqq|z|<2 a$. For $j<l(k)$ we have

$$
N^{-j} \log \delta \leqq N^{-j} \log \left|Q \circ R_{f}\left(w_{k}\right)\right| \leqq N^{-j} \log \gamma
$$

where

$$
\delta=\inf \{|Q(z)|:|z|<a\}>0 \text { and } \gamma=\sup \{|Q(z)|:|z|<a\}<\infty .
$$

For $j \geqq l(k)$,

$$
\begin{aligned}
0 & \leqq N^{-j} \log \left|Q \circ R_{j}\left(w_{k}\right)\right| \leqq N^{-j}\left(\log b+M \log \left|R_{j}\left(w_{k}\right)\right|\right) \\
& \leqq N^{-j}\left(\log b+M \log \left|R_{j-l}\left(R_{l}\left(w_{k}\right)\right)\right|\right) \\
& \leqq N^{-j}\left(\log b+M\left(\left(L+\cdots+L^{j-l}\right) \log b+L^{j-l} \log \left|R_{l}\left(w_{k}\right)\right|\right)\right) \\
& \leqq N^{-j}\left(\log b+M\left(\left(L+\cdots+L^{j-l}\right) \log b+L^{j-l} \log (2 a)\right)\right) \\
& \leqq K(L / N)^{j} .
\end{aligned}
$$


Thus the limit above can be taken term by term, and the formula for the logarithmic potential is justified.

We can now obtain the formula for $I\left(\mu_{\zeta}\right)$ for any $\zeta$ in $C$. Using the Fubini and dominated convergence theorems

$$
\begin{aligned}
I\left(\mu_{\zeta}\right) & =\iint \log \frac{1}{|z-w|} d \mu_{\zeta}(z) d \mu_{\zeta}(w)=\int S(w) d \mu_{\zeta}(w) \\
& =-\frac{1}{N} \int \sum_{j=0}^{\infty} N^{-j} \log \left|Q \circ R_{j}(w)\right| d \mu_{\zeta}(w) \\
& =-\frac{1}{N}\left(\sum_{j=0}^{\infty} N^{-j}\right) \int \log |Q(w)| d \mu_{\zeta}(w) \\
& =\frac{1}{1-N} \int \log |Q(w)| d \mu_{\zeta}(w) \\
& =\frac{1}{1-N} \int A \log |Q(w)| d \mu_{\zeta}(w) \\
& =\frac{1}{1-N} \log |c|+\frac{1}{N(1-N)} \sum_{j=1}^{M} \log \left|P\left(q_{j}\right)\right| .
\end{aligned}
$$

We shall next show the uniqueness of $\mu_{\zeta}$ for all $\zeta$ in $C$. Let $\zeta_{1}$ and $\zeta_{2}$ be in $C$. Let $\sigma=\mu_{\zeta_{1}}-\mu_{\zeta_{2}} . I(|\sigma|) \leqq I\left(\mu_{\zeta_{1}}-\mu_{\zeta_{2}}\right)<\infty$. Now using Fubini's Theorem again

$$
\begin{aligned}
I(\sigma) & =\int\left(\int \log |z-w|^{-1}\right) d\left(\mu_{\zeta_{1}}(z)-\mu_{\zeta_{2}}(z)\right) d \sigma(w) \\
& =\int(S(w)-S(w)) d \sigma(w)=0 .
\end{aligned}
$$

So, by Theorem 16.42 of Hille [3, p. 241], $\mu_{\zeta_{1}}=\mu_{\zeta_{2}}$. Since $\zeta_{1}$ and $\zeta_{2}$ are arbitrary in $C$, we have shown that all $\zeta$ in $C$ produce identical $\mu_{\zeta}$. Finally, by Lemma 7 , if $\mu_{\zeta}=\mu$ for all $\zeta$ in $C$, and if $\nu$ is any nonatomic $A$-invariant probability measure then

$$
\int f d \nu=\int\left(\int f d \mu_{\zeta}\right) d \nu=\int f d \mu
$$

for all continuous $f$, so $\nu=\mu$.

THEOREM 5. For $R(z)=P(z) / Q(z)$ with $P$ a monic polynomial and $Q$ a polynomial such that the degree of $P \geqq$ the degree of $Q+2, \mu$ is ergodic with respect to the transformation $R$ where $\mu$ is the unique A-invariant nonatomic probability measure.

Proof. If $R^{-1}(B)=B$ a.e. and $B$ is a Borel set, then for any Borel measurable $f$ in $L_{1}$,

$$
\begin{aligned}
\int \chi_{B} f d \mu & =\int\left(\frac{1}{n+1} \sum_{k=0}^{n} \chi_{B} \circ R_{k}\right) f d \mu \\
& =\int \chi_{B}\left(\frac{1}{n+1} \sum_{k=0}^{n} A^{k} f\right) d \mu \rightarrow \int f d \mu \int \chi_{B} d \mu
\end{aligned}
$$

i.e., $\chi_{B}$ is a constant a.e. 
In general, for compact $E \subset C$ either $I(v)$ is infinite for all probability measures supported on $E$, or there exists a unique $\nu_{0}$, called the equilibrium measure, which minimizes $I(\nu)$. In this case the capacity $C$ of $E$ is defined by $C=\exp \left[-I\left(\nu_{0}\right)\right]$. The above facts can be found in Hille [3]. Brolin [1] proved that $\mu$ is the equilibrium measure when $R$ is a polynomial.

We will write $\|f\|_{F}$ for the uniform norm of $f$ on $F$; i.e.,

$$
f \|_{F}=\sup \{|f(z)|: z \in F\}
$$

THEOREM 6. We have

$$
\exp \left(\frac{1}{N-1} \int \log |Q| d \mu\right) \leqq C \leqq\|Q\|_{F}^{1 /(N-1)}
$$

$\mu$ is the equilibrium measure if and only if $Q$ is constant; i.e., $R$ is a polynomial, and in this case $C=Q^{1 /(N-1)}$.

Proof. Let $v$ be the equilibrium measure. Then

$$
\log C=-I(\nu) \geqq-I(\mu)=\frac{1}{N-1} \int \log |Q| d \mu,
$$

or

$$
\exp \left(\frac{1}{N-1} \int \log |Q| d \mu\right) \leqq C
$$

We also have $C=\lim _{k \rightarrow \infty}\left(\left\|T_{k}\right\|_{F}\right)^{1 / k}$ where $T_{k}$ is the $k$ th Tchebycheff polynomial for $F$, i.e., the monic polynomial of degree $k$ with minimum $\left\|T_{k}\right\|_{F}$. Since $R(F) \subset F$,

$$
\left\|T_{n N}\right\|_{F}^{1 / n N} \leqq\left\|Q^{n} T_{n} \circ R\right\|_{F}^{1 / n N} \leqq\|Q\|_{F}^{1 / N}\left\|T_{n}\right\|_{F}^{1 / n N}
$$

Taking limits on $n$ we get $C \leqq\|Q\|_{F}^{1 / N} C^{1 / N}$ from which the desired inequality follows.

If $Q$ is constant we have

$$
C=|Q|^{1 /(N-1)}=\exp (-I(\mu)) .
$$

On the other hand, if $\mu$ is the equilibrium measure then $u$ is constant almost everywhere on $F$ so that

$$
\begin{aligned}
u(w) & =u(R(w))=-\frac{1}{N} \sum_{j=0}^{\infty} N^{-j} \log \left|Q \circ R_{j+1}(w)\right| \\
& =N u(w)+\log |Q|(w),
\end{aligned}
$$

and $Q$ must be constant. 


\section{REFERENCES}

1. Hans Brolin, Invariant sets under iteration of rational functions, Ark. Mat. 6 (1965), 103144. MR 33 \#2805.

2. P. Fatou. Sur les équations fonctionnelles, Bull. Soc. Math. France 47 (1919), 161-271.

3. Einar Hille, Analytic function theory, Vol. 2, Introductions to Higher Math., Ginn, Boston, Mass., 1962. MR 34 \#1490.

4. G. Julia, Memoire sur l'itération des fonctions rationnelles, J. Math. Pures Appl. (8) 1 (1918), 47-245.

Department of Mathematics, University of Hawail, Honolulu, Hawail 96822

Current address (Oba): Department of Mathematics, Loyola University, Los Angeles, California 90045 